\title{
Bystander Privacy in Lifelogging
}

\author{
Soumyadeb Chowdhury \\ Singapore Institute of Technology \\ Soum.Chowdhury@singaporetech.edu.sg \\ Md Sadek Ferdous \\ University of Southampton \\ S.Ferdous@soton.ac.uk
}

\author{
Joemon M Jose \\ University of Glasgow \\ Joemon.Jose@glasgow.ac.uk
}

\begin{abstract}
Lifelogging, a technique for digitally gathering every moment in life using wearable cameras, is a growing phenomenon. However, it is important to remember that in addition to recording their own lives, users are recording others as well, which raises privacy issues around lifelogging from a bystander's perspective. This warrants conducting bystander-focused privacy studies in an uncontrolled environment, by reaping upon the experience of practitioners from multiple domains, but not limited to, urban science, sociology, privacy, usability, psychology, and law, to design such studies.
\end{abstract}

Lifelogging, Privacy, Bystander, Experiment Design, Society

\section{INTRODUCTION}

Lifelogging wearable cameras such as Autographer (Autographer, 2009) and Narrative Clip (Narrative clip, 2012) passively capture photos at a very high rate- Autographer (100-200 images per hour) and Narrative Clip (120 images per hour). These photographs consist of comprehensive records of users' daily activities (Gurrin, 2014). However, it is important to note that in addition to recording their own lives, users are recording others as well. This intrusive and invasive nature of lifelogging has understandably drawn the attention of advocates of personal privacy in different domains. The photos may be captured in different settings/scenarios including, but not limited to private (living room), intimate (time spent with loved ones), corporate (workplace). Hence it is necessary to understand the privacy implications of the lifelog images from the perspectives of both the lifeloggers (LLs, i.e. person wearing the device) and bystanders (BSs i.e. known or unknown people captured in the photographs). The use of wearable cameras thus raise serious questions of permission from the BSs, especially if the photos are eventually published.

To the best of our knowledge, none of the existing studies have focused on the privacy of the bystanders. Some practitioners have suggested that the privacy of the lifeloggers is more important than that of bystanders (Lifelogging, 2016). However, there are no research evidence to support such a suggestion. The right to privacy is one of the fundamental human rights in any modern society. It advocates and facilitates mechanisms to uphold the privacy of all individuals within the society. However, what is private is highly debated because there are social, legal, political, technical and psychological connotations to privacy (Gurrin et al., 2014).

In this paper, we contend that it is necessary to conduct longitudinal lifelogging user studies in the natural environment of the LLs to better understand the privacy concerns raised by the BSs. Our contention is based upon the anecdotal evidence gathered from a study conducted in the United Kingdom (Glasgow), where a number of BSs (who were either known or unknown to the LLs) had raised privacy concerns. This evidence might be considered a bit conflicting compared to the results reported in (Hoyle et al., 2014), but it clearly demonstrates a knowledge gap in the context of lifelogging privacy, which warrants a systematic investigation.

\section{RELATED STUDIES}

From a legal perspective, Allen (2008) has suggested that lifelogs should not be recorded without the explicit permission of the bystanders, and has proposed a counter-technology to block lifelog surveillance, but did not provide any guidance on how to implement such a technology. Kelly et al. (2013) have suggested that privacy and anonymity of third parties must be protected and image containing third parties should not be published without their prior consent. The most recent work reported in Hoyle et al. (2014 and 2015) have studied the reactions of the bystanders from a LL's perspective. The study demonstrated that 26 out of 36 LLs reported positive interest from the BSs, and none of the LLs reported to have encountered opposition from the BSs. We believe that these results are likely to vary for each study, and may depend upon the surroundings (when and 
where a wearable device is used) and the frequency of use. For example, there is a difference between using the device in solitude, among a group of classmates (in class), group of strangers, or inside a business seminar. We also contend that perceptions about privacy infringements is likely to vary from one individual to another, individual across different age groups, and privacy laws enforced in a country.

\section{EVALUATION}

We recruited 40 LLs (subjects in this case) by sending emails to the university mailing list, once ethics approval was granted by the ethics committee to conduct the study. The LLs were asked to use a wearable camera, "Autographer", for a period of 3 days (6-8 hours every day). The devices were handed over for a week, to give some slack time to the LLs to get used to the devices. LLs were also provided with an instruction sheet to help them use the devices. Additionally, a list of suggestions were provided in order to reduce the potential risks arising from our study. The suggestions included, but were not limited to: (1) avoiding using the device in rest rooms and in places where photography may be prohibited; (2) if a bystander enquires about the device, first the image capture must be paused, and then the objectives of our study must be explained; (3) if a bystander seems to be concerned, then step 2 should be followed and they should be provided with our contact information, as well as a URL containing further details about our project. The LLS were also asked to note down the date and time of the incident in a deletion card, so that we were able to delete the images captured for that period of time. This framework ensured that the study reflected the way these devices will be actually used in real-life.

After the study, semi-structured interviews were conducted with the LLs to understand the reactions of the bystanders. A majority of the LLs participating in our study (29 out of 40) had reported that people they lived with, i.e. either their family members or flatmates enquired about the device (in particular about characteristics of the device). A majority (i.e. around $85 \%$ ) of the flatmates, and family members were not in favour of using the device inside the shared flat. They were concerned about: how the lifelogs will portray themselves; location where the contents will be stored; how the contents will be used; what sort of activities will be captured, especially when the device is either used in the private space or homely environment. 18 LLs had reported that their colleagues in the workplace enquired about the device, and requested the LLs to avoid using the device during office hours. There were 9 instances, where the line manager explicitly asked the LLs to avoid using the lifelogging cameras during the office hours, and in the business gatherings, though there were no policies set by the organization to prevent people from taking pictures, during office hours (according to the LLs). It was also reported that 12 bystanders (unknown people in public transport, and shopping stores), enquired about the device, and showed discontent and concern after hearing its characteristics. The bystanders politely requested the lifeloggers to delete their photographs, because they did not like to the idea of being randomly photographed. These bystanders also told that they felt as if they were being tracked. The LLs also pointed out that the attitude of the people towards privacy seemed to depend upon: (1) the ability to identify the camera on the body (i.e. visibility of the device); (2) what sort of information about the wearable is provided during the enquiry, and how this information is described. Thus the experimental protocol used for a study may also influence the perception of the public. In summary, the bystanders were concerned about: (1) self-impression management, how they are being represented in the lifelogs, (2) being tracked, i.e. identified being at a certain place engaged in certain activities; (3) how the lifelogs can be changed to give a negative impression about them (or activities).

\section{CONCLUSION}

The recent advances in pervasive sensing, especially wearable cameras have raised privacy concerns, especially from the perspectives of the BSs. We do not claim that our study with LLs is rigorous, but contend that the results underscore the need for further studies with BSs to understand their concerns. But in order to conduct the studies, and make them comprehensive, and comparable, it is necessary to establish an evaluation protocol comprising of a set of common guidelines. This set of guidelines would need to address issues such as: consistent way of designing experiments; protocols to be used, i.e. minimum duration of study, metric to be reported, interpretation of metrics; type of training to be given before starting the experiment, where to place the device on the body; instructions for the LLs during exigencies. This will help to establish a common set of criteria that can be used to compare the results obtained in a study and demonstrate its viability in a specific context. Practitioners and experts from multiple domains such as security, legal, privacy, urban sciences, and psychology, should come together to establish rules for effective experimental design to conduct lifelogging privacy studies, which can be adopted in the future. By taking such steps, we can: (1) edge closer to design and development of a privacy-preserving lifelogging system that protects the privacy as well security of the 
bystanders; (2) understand the societal and urban aspects negatively influencing the acceptance of such technologies.

\section{ACKNOWLEDGEMENT}

The authors acknowledge support from Integrated Multimedia City Data (iMCD), a project within the ESRC-funded Urban Big Data Centre (ES/L011921/1).

\section{REFERENCES}

Allen, A., (2008). Dredging up the past: Lifelogging memory, and surveillance. The University of Chicago Law Review, 75(1). 47-74.

Autographer. (2009) The World's First Intelligent Wearable Camera, OMG Life Ltd Available from: http://www.autographer.com (Accessed: 20 May 2016).

Gurrin, C., Smeaton, A.F. and Doherty, A.R., (2014). 'LifeLogging: Personal Big Data. Foundations and trends in information retrieval, 8(1).1-125.
Gurrin, C., Albatal, R., Joho, H. and Ishii, K., (2014). A privacy by design approach to lifelogging. Digital Enlightenment Yearbook 2014: Social Networks and Social Machines, Surveillance and Empowerment. 49.

Hoyle, R, et al., (2014), September. Privacy behaviors of lifeloggers using wearable cameras. In Proceedings of the 2014 ACM International Joint Conference on Pervasive and Ubiquitous Computing. 571-582.

Hoyle, R, et al., (2015), April. Sensitive Lifelogs: A Privacy Analysis of Photos from Wearable Cameras. In Proceedings of the 33rd Annual ACM Conference on Human Factors in Computing Systems. 1645-1648.

Kelly, P, et al., (2013). An ethical framework for automated, wearable cameras in health behavior research. American journal of preventive medicine, 44(3). 314-319.

Lifelogging: The end of privacy - Part 3. (2016). https://e27.co/lifelogging-the-end-of-privacy-part3-20141017/. Accessed: 2016- 03- 18.

Narrative clip 2. (2012). Memoto AB Available at: http://getnarrative.com (Accessed: 23 May 2016). 